\title{
Chinese Character Recognition by Tchebichef Moment Features
}

\author{
Bing Hu and Simon Liao, Member, IACSIT
}

\begin{abstract}
Moment descriptors have long been applied in object recognition since the early years of the development of the moment theories. Nowadays, discrete orthogonal moments have been studied and proposed for they are superior to traditional continuous ones. In this paper, a set of moment features extracted from the discrete Tchebichef moments for Chinese character recognition is presented. A new method of evaluating the variance values of each moment feature is applied in this research. Tested on a set of 6,763 Chinese characters, our newly proposed Tchebichef moment features perform very well in distinguishing all Chinese character pairs that have similar structures.
\end{abstract}

Index Terms-Discrete orthogonal moments, tchebichef moments, Chinese character recognition.

\section{INTRODUCTION}

Since $\mathrm{Hu}$ [1] introduced the moment methods in 1961, moment descriptors have been widely used in image representation, pattern recognition, and object classification. One of the applications using moment features is the Chinese character recognition. Chinese characters are very different from many other languages in conveying information, while the structure of a character is a key to its meaning and pronunciation. Many feature extraction methods applied in the Chinese character recognition systems are based on the local features, such as strokes and feature points [2]-[5]. While the existing methods are quite efficient in general, there are some difficulties to distinguish two characters when they have very close structures. On the other hand, the moment method has the advantage of utilizing the global features of the Chinese characters, therefore, it can be used as a complementary scheme to overcome the obstacles confronted by other systems. Some Chinese character recognition systems based on the orthogonal moment descriptors have been reported [6]-[8]. Recently, the emergence of discrete orthogonal moments has substantially enriched the moment methods. Moments based on Tchebichef and Krawtchouk polynomials were introduced by Mukundan et al. [9] and Yap et al. [10] in 2001 and 2003, respectively. Since the computation of discrete orthogonal moments is not involved with numerical approximation of the weight function, while maintaining the property of orthogonal, the discrete moments are superior to traditional

Manuscript received June 12, 2013; revised July 30, 2013

Bing Hu is with the Applied Computer Science Department, University of Winnipeg, Manitoba, Canada (e-mail: hu-b86@webmail.uwinnipeg.ca).

Simon Liao is with the Applied Computer Science Department, University of Winnipeg, Manitoba, Canada (e-mail: s.liao@.uwinnipeg.ca). continuous orthogonal moments in terms of calculation accuracy and speed. Since they were introduced, the discrete orthogonal moments have been productively utilized in image processing [11]-[13], pattern recognition [14], [15], and many other scientific field [16]. In this research, we have utilized the Tchebichef moments in the aspect of Chinese character recognition. Four lower orders Tchebichef moments with the highest variance values are selected as features in the four dimensional Tchebichef moment space. A set of 6,763 Chinese characters, which are defined in the Chinese standard GB2312, is used as the testing characters. Our results show that the recognition ability of the four features based on the Tchebichef moments overwhelms the ones utilizing continuous orthogonal moments.

\section{TCHEBICHEF MOMENTS}

The definition of the $n$-th order classical Tchebichef Polynomial is defined as

$$
t_{n}(x)=(1-N)_{n 3} F_{2}(-n,-x, 1+n ; 1,1-N ; 1)
$$

where $x, n=0,1,2, \ldots, N, N>0, p \in(0,1) \cdot{ }_{2} F_{1}$ is the hypergeometric function. $a_{k}$ is the Pochhammer symbol given by

$$
\begin{gathered}
(a)_{k}=a(a+1) \ldots(a+k-1)=\frac{\Gamma(a+k)}{\Gamma(a)} \\
{ }_{2} F_{1}(a, b ; c ; z)=\sum_{k=0}^{\infty} \frac{(a)_{k}(b)_{k}}{(c)_{k}} \frac{z^{k}}{k !}
\end{gathered}
$$

With the definitions above, (1) can also be written as

$$
t_{n}(x)=n ! \sum_{k=0}^{n}(-1)^{n-k}\left(\begin{array}{c}
N-1-k \\
n-k
\end{array}\right)\left(\begin{array}{c}
n+k \\
n
\end{array}\right)\left(\begin{array}{l}
x \\
k
\end{array}\right)
$$

The scaled Tchebichef polynomials are defined as

$$
t_{n}(x)=\frac{t_{n}(x)}{\beta(n, N)}
$$

where $t_{n}(x)$ is the discrete Tchebichef polynomial given by (1), and $\beta(n, N)$ is a constant which is independent of $x$. Under the discussion above, the squared norm of the scaled polynomial is given by

$$
p(n, N)=\frac{\rho(n, N)}{\beta(n, N)^{2}}
$$

where $\rho(n, N)$ is 


$$
\begin{aligned}
\rho(n, N) & =\frac{N\left(N^{2}-1\right)\left(N^{2}-2^{2}\right) \ldots\left(N^{2}-n^{2}\right)}{2 n+1} \\
& =(2 n) !\left(\begin{array}{c}
N+n \\
2 n+1
\end{array}\right), n=0,1, \ldots, N-1
\end{aligned}
$$

Which satisfies the orthogonal condition

$$
\begin{aligned}
\sum_{x=0}^{N-1} t_{m}(x) t_{n}(x)= & \rho(n, N) \delta_{m n}, \\
& 0 \leq m, n \leq N-1
\end{aligned}
$$

The scale factor $\beta(n, N)$ is usually a function of $N$ which grows at the speed of $N^{n}$. The simplest form of $\beta(n, N)$ can be

$$
\beta(n, N)=N^{n}
$$

To make the scaled polynomial set orthogonal, $\beta(n, N)$ is given by

$$
\beta(n, N)=\sqrt{\rho(n, N)}
$$

then we define the Tchebichef moment as

$$
\begin{array}{r}
T_{p q}=\frac{1}{p(p, N) p(q, N)} \sum_{x=0}^{N-1} \sum_{y=0}^{N-1} t_{p}(x) t_{p}(y) f(x, y) \\
p, q=0,1,2, \ldots, N-1
\end{array}
$$

One method being used commonly to make computation of moments less demanding for the processor is the recurrence relation. The three-term recurrence relation of the weighted Tchebichef Polynomial with respect to $n$ is defined as

$$
\begin{aligned}
& (n+1) t_{n+1}(x)-(2 n+1)(2 x-N+1) t_{n}(x) \\
& \quad+n\left(N^{2}-n^{2}\right) t_{n-1}(x)=0, n=1,2 \ldots, N-1
\end{aligned}
$$

\section{Chinese ChARACTER RECOGNITION}

Our primary task is to find efficient lower order Tchebichef moment feature functions to distinguish Chinese characters more effectively. In the view of statistics, moments with higher variance values have more power to differentiate objects. In order to select the best Tchebichef moment descriptors, we firstly calculated the variance values of lower order Tchebichef moments of a set of 6,763 Chinese characters defined in China's national standard GB2312, with the font of song. All of these Chinese characters are binary images with a size of $24 \times 24$.

TABLE I: VARIANCE VALUES OF LOWER ORDER TCHEBICHEF MOMENTS $T_{M N}$

\begin{tabular}{ccccc}
\hline \hline & 0 & 1 & 2 & 3 \\
\hline 0 & 0.7964 & 0.3664 & 0.8670 & 0.6905 \\
1 & 0.2426 & 0.2083 & 0.3496 & 0.3042 \\
2 & 0.4722 & 0.2643 & 0.4720 & 0.3962 \\
3 & 0.2317 & 0.2038 & 0.3450 & 0.3247 \\
\hline \hline
\end{tabular}

Based on the variance values of 16 lower order Tchebichef moments shown in Table I, we have selected the highest four variance values as our four-dimensional Tchebichef moment feature vector

$$
\mathbf{V}\left[\mathbf{f}_{1}=T_{00}, \mathbf{f}_{2}=T_{02} \mathbf{f}_{3}=T_{03} \mathbf{f}_{4}=T_{20}\right] \sqrt{a^{2}+b^{2}}
$$

To measure the distance between any pair of Chinese characters in our four-dimensional Tchebichef moment

\begin{tabular}{|c|c|c|c|}
\hline \multicolumn{4}{|c|}{ DISTANCE } \\
\hline & 祯玎 & 沪耕 & 伟佴 \\
\hline$d_{i j}(T)$ & 1.9425 & 2.3142 & 2.0167 \\
\hline \multirow[t]{2}{*}{$d_{i j}(L)$} & 0.2436 & 0.3946 & 0.2758 \\
\hline & 危笡 & 㬝蹉 & 锂蛤 \\
\hline$d_{i j}(T)$ & 1.5746 & 1.6748 & 2.0435 \\
\hline \multirow[t]{2}{*}{$d_{i j}(L)$} & 0.1754 & 0.3241 & 0.3157 \\
\hline & 恐醒 & 冥蜓 & 胛籁 \\
\hline$d_{i j}(T)$ & 1.8564 & 1.7493 & 1.3465 \\
\hline \multirow[t]{2}{*}{$d_{i j}(L)$} & 0.1564 & 0.1443 & 0.1647 \\
\hline & 祥砑 & & \\
\hline$d_{i j}(T)$ & 1.6984 & & \\
\hline$d_{i j}(L)$ & 0.2546 & & \\
\hline
\end{tabular}
feature space, the Euclidean distance is adopted as our measurement

$$
d\left(\mathbf{f}_{i}, \mathbf{f}_{j}\right)=\sqrt{\left(f_{1 i}-f_{1 j}\right)^{2}+\left(f_{2 i}-f_{2 j}\right)^{2}+\left(f_{3 i}-f_{3 j}\right)^{2}+\left(f_{4 i}-f_{4 j}\right)^{2}}
$$

where $\mathbf{i}$ and $\mathbf{j}$ represent any two Chinese characters, $i, j \in[1,6763]$.

In our experiment, the distances between all pairs of Chinese characters, $(6763 \times 6762) / 2=22,865,703$ in total, are calculated.

TABLE II: THE TEN PAIRS OF CHINESE CHARACTERS WITH THE CLOSEST

For comparison, we have formed a four-dimensional Legendre moment vector with the same selection process leading to (13). Table II shows the ten pairs of Chinese characters that have the closest distances in the four-dimensional Tchebichef moment space, compared by their distances in the Legendre moment vector space.

The $(p+q)$ th order Legendre moment is defined as:

$$
L_{p q}=\frac{(2 p+1)(2 q+1)}{4} \int_{-1}^{1} \int_{-1}^{1} P_{p}(x) P_{q}(y) f(x, y) d x d y
$$

where $P_{p}(x)$ is a Legendre polynomial of order $p$, which is defined as:

$$
P_{p}(x)=\sum_{j=0}^{p} C_{p j} x^{j}
$$

And

$$
C_{p j}= \begin{cases}(-1)^{(p-j) / 2} \frac{1}{2^{p}} \frac{(p+j) !}{[(p-j) / 2] ![(p+j) / 2] ! j !}, & \text { if } p-j \text { is even } \\ 0, & \text { if } p-j \text { is odd }\end{cases}
$$

Legendre polynomials form a complete orthogonal basis within the range of $[-1,1]$. 
TABLE III: DistANCES ON SOME CHINESE CHARACTERS PAIRS WHICH ARE Close in STRuCTURES AND ARE DifFicult to Be ReCOGNIZED By SOME

\begin{tabular}{|c|c|c|c|}
\hline \multicolumn{4}{|c|}{ SYSTEMS } \\
\hline & 粘钻 & 借惜 & 诽排 \\
\hline$d_{i j}(T)$ & 8.5463 & 8.6748 & 9.0346 \\
\hline \multirow[t]{2}{*}{$d_{i j}(G)$} & 1.8629 & 2.1676 & 4.2597 \\
\hline & 扬杨 & 倍培 & 人入 \\
\hline$d_{i j}(T)$ & 7.5786 & 8.3564 & 8.9785 \\
\hline \multirow{2}{*}{$d_{i j}(G)$} & 3.5059 & 2.8710 & 2.4462 \\
\hline & 婏婏 & 千于 & 大太 \\
\hline$d_{i j}(T)$ & 8.6947 & 7.9836 & 7.5492 \\
\hline \multirow[t]{2}{*}{$d_{i j}(G)$} & 2.0407 & 4.7123 & 3.3850 \\
\hline & 大犬 & 末末 & 观现 \\
\hline$d_{i j}(T)$ & 8.3154 & 6.9827 & 6.8749 \\
\hline \multirow[t]{2}{*}{$d_{i j}(G)$} & 5.5103 & 0.7676 & 2.9021 \\
\hline & 沙抄 & 垃拉 & 如知 \\
\hline$d_{i j}(T)$ & 8.0645 & 8.1674 & 7.2957 \\
\hline \multirow[t]{2}{*}{$d_{i j}(G)$} & 1.7538 & 2.9099 & 4.8679 \\
\hline & 借惜 & 拒柜 & 扑朴 \\
\hline$d_{i j}(T)$ & 7.8347 & 8.2495 & 7.6948 \\
\hline \multirow[t]{2}{*}{$d_{i j}(G)$} & 2.1676 & 3.3237 & 2.4950 \\
\hline & 惜错 & 诗待 & 听昕 \\
\hline$d_{i j}(T)$ & 9.3165 & 9.0442 & 8,5664 \\
\hline \multirow[t]{2}{*}{$d_{i j}(G)$} & 3.2325 & 1.3912 & 2.1927 \\
\hline & 尸户 & 天夭 & 木术 \\
\hline$d_{i j}(T)$ & 8.5463 & 8.6748 & 9.0346 \\
\hline \multirow[t]{2}{*}{$d_{i j}(G)$} & 3.3113 & 2.0222 & 0.7212 \\
\hline & 王玉 & 王主 & 论沦 \\
\hline$d_{i j}(T)$ & 7.5786 & 8.3564 & 8.9785 \\
\hline \multirow[t]{2}{*}{$d_{i j}(G)$} & 0.6923 & 2.4831 & 4.7783 \\
\hline & 今令 & 茶茶 & 伦抡 \\
\hline$d_{i j}(T)$ & 8.6947 & 7.9836 & 6.5814 \\
\hline \multirow[t]{2}{*}{$d_{i j}(G)$} & 1.2917 & 1.7076 & 1.7568 \\
\hline & 日曰 & 夫失 & 井并 \\
\hline$d_{i j}(T)$ & 8.3154 & 6.9827 & 6.8749 \\
\hline \multirow[t]{2}{*}{$d_{i j}(G)$} & 1.1453 & 1.3932 & 2.4308 \\
\hline & 冊母 & 万方 & 竟竟 \\
\hline$d_{i j}(T)$ & 8.0645 & 8.1674 & 7.2957 \\
\hline \multirow[t]{2}{*}{$d_{i j}(G)$} & 1.6591 & 1.7066 & 0.4604 \\
\hline & 怕帕 & 奖浆 & 捡俭 \\
\hline$d_{i j}(T)$ & 7.8347 & 8.2495 & 7.6948 \\
\hline$d_{i j}(G)$ & 0.8103 & 5.2217 & 4.4499 \\
\hline
\end{tabular}

The results shown in Table II indicate that by using the discrete Tchebichef moment vector, every pair in the set of 6,763 Chinese characters can be distinguished significantly.
We would like to mention that all of the ten closest pairs characters may be recognized without difficulty by other existing Chinese character recognition systems.

One of the most challenging tasks for Chinese characters recognition is to recognize some of Chinese characters that share similar structures. To address this issue, we have selected a set of Chinese character pairs which are difficult to be distinguished by other systems.

$d_{i j}(T)$ measures the distance using discrete Tchebichef moments method while $d_{i j}(G)$ measures the distance using orthogonal Gegenbauer moments method[17].

The results shown in Table III indicate that the four-dimensional Tchebichef moment based vector perform very well for all of these Chinese character pairs.

\section{CONCLUSION}

A Chinese character recognition system with the Tchebichef moment-based method is described in this paper. Four Tchebichef moments with the largest variance values are selected to form a four-dimensional feature vector for the system. A set of 6,763 Chinese characters is used as the testing set, with each character has the size of $24 \times 24$ pixels. The results show that by using Tchebichef moment-based features, each pair of the testing Chinese characters can be distinguished significantly.

For the ten pairs of Chinese characters which are most difficult to be distinguished with the proposed system, they have very different structures and can be recognized by other existing system efficiently. On the other hand, some Chinese characters pairs with very similar structures can be easily recognized by our system. Therefore, our proposed Tchebichef moment-based recognition system can be applied as a complementary scheme with other Chinese character recognition systems to increase the successful recognition rate.

\section{REFERENCES}

[1] M. K. Hu, "Visual problem recognition by moment invariant," IRE Trans.Inform.Theory, vol. IT-8, pp. 179-187, Feb 1962.

[2] S. Dudani, K. Breeding, and R. McGhee, "Aircraft identification by moment invariants," IEEE Trans. Comput, vol. 26, pp. 39-45, Feb.1977.

[3] D. Casasent and R. Cheatham, "Image segmentation and real image tests for an optical moment-Based feature extractor," Opt. Commun., vol. 51, pp. 227-230, Sept. 1984.

[4] M. R. Teague, "Image analysis via the general theory of moments," $J$. Opt. Soc. Amer., vol. 70, pp. 920-930, Aug. 1962.

[5] K. W. Gan and K. T. Lua, "A new approach to stroke and feature point extraction in Chinese character recognition," Pattern Recognition Letter, vol. 12, pp. 381-387, 1991.

[6] J. Liu and S. P. Ma, "An overview of printed Chinese character recognition techniques," in Proc. the International Conference on Chinese Computing '96, Singapore, June 4-7, 1996, pp. 325-333.

[7] X. Z. Zhang, C. D. Yan, and X. Y. Liu, "Feature point method of Chinese character recognition and its application," Journal of Computer Science, vol. 5, no. 4, 1990.

[8] P. T. Yap and R. Paramesran, "Image analysis by krawtcouk moments," IEEE Trans. On Image Processing, vol. 12, no.11 pp. 1367-1377, November 2003.

[9] R. Mukundan, S. H. Ong, and P. A. Lee, "Image analysis by Tchebichef moments," IEEE Trans. Image Processing, vol. 10, pp. 1357-1364, Sept. 2001.

[10] H. Q. Zhu et al., "Image analysis by discrete orthogonal Racah moments," Signal Processing, vol. 87, no. 4, pp. 687-708, 2007. 
[11] G. Faouzi et al., "Image reconstruction from a complete set of similarity invariants extracted from complex moments," Pattern recognition letters, vol. 27, no. 12, pp. 1361-1369, 2006.

[12] P. T. Yap, R. Paramesran, and S. H. Ong, "Image analysis using hahn moments." Pattern Analysis and Machine Intelligence, vol. 29, no. 11, pp. 2057-2062, 2007

[13] R. C. Zhi and Q. Q. Ruan, "A comparative study on region-Based moments for facial expression recognition," International Proceedings of Image and Signal Processing, vol. 2, 2008.

[14] A. J. Nor'aini and P. Raveendran, "Improving face recognition using combination of global and local features," International Proceedings of Mechatronics and its Applications, 2009.

[15] M. Alghoniemy and A. H. Tewfik, "Image watermarking by moment Invariants," in Proc. IEEE Int. Conf. Image Processing, 2000, pp.73-76.

[16] X. Pan, Q. H. Chen, and Z. Liu, "Content-Based 3-D retrieval by krawtchouk moments," Image Analysis and Recognition Lecture Notes in Computer Science, vol. 4142, pp. 217-224, 2006.
[17] S. Liao et al., "Chinese character recognition via gegenbauer moments," Pattern Recognition, vol. 3, 2002.

Bing Hu received the BSc degree in 2011 from Shanghai Jiaotong University, Shanghai, China and is now studying as a Master student under the supervision of professor Simon Liao in the University of Winnipeg, Manitoba, Canada.

Simon X. Liao received the BSc degree in 1982 from Beijing Normal University, Beijing, China, and the MSc and $\mathrm{PhD}$ degrees in electrical and computer engineering from the University of Manitoba, Winnipeg, Canada, in 1988 and 1993, respectively.

Dr. Liao has been with the University of Winnipeg, Winnipeg, Canada, since 1991. He is currently a professor in the Department of Applied Computer Science. His research interests include pattern recognition, computer vision, and image processing. 